Resumen: Cada vez cobran más popularidad los productos que incluyen técnicas artesanales en sus procesos de producción, no solo por la experiencia que le brindan al consumidor, también por la creciente conciencia social y ambiental que está en auge y que motiva la compra de propuestas con el valor que solo los productos hechos a mano pueden brindar. Esto presenta una invaluable oportunidad para todos los maestros artesanos con los que cuenta América Latina. Si bien es cierto que tiempo atrás se fueron perdiendo sus técnicas ancestrales, es ahora cuando se presenta la oportunidad ideal para su recuperación. Sin embargo, hay conocimientos y estrategias de difícil acceso para estas comunidades de artesanos, los cuales si son bien implementados pueden incrementar considerablemente la competitividad de los productos. Estos conocimientos pueden ser encontrados a través de los diseñadores e investigadores, quienes tienen el acceso, la formación y la capacidad de trasladar la información a los artesanos de tal forma que las propuestas generadas sean de aún más valor, con el potencial de penetrar el mercado global.

Palabras clave: Diseño - Artesanía - Diseño colaborativo - Local - Global.

[Resúmenes en inglés y portugués en la página 164]

(1) Licenciatura en Diseño Industrial de la Universidad Rafael Landívar, Investigadora académica y Coordinadora del Centro de Atención a la MIPYME en la misma universidad, a través del Instituto de Investigación y Estudios Superiores en Arquitectura y Diseño (Indis).

\title{
La pandemia
}

La actual pandemia no solo trae consigo una crisis sanitaria de una magnitud inconmensurable, dados los confinamientos y el distanciamiento social al que todo el mundo se ha tenido que regir es difícil calcular el impacto económico que desde ya está ocasionando. El 
Fondo Monetario Internacional (FMI) estima que al menos 170 naciones miembro verán crecimientos negativos en sus ingresos per cápita, siendo los más afectados los países en desarrollo y los mercados emergentes (BBC, 2020). Esta misma organización pronostica que la economía global se contraerá alrededor de un 3\% durante el 2020. Sin embargo, Latinoamérica sufrirá un descenso aún mayor, hasta ahora han estimado que será del $5.3 \%$ para el mismo período, lo cual es comparable a la contracción económica que sufrió la región durante la Gran Depresión de 1930 (CEPAL, 2020).

Es importante tomar en cuenta que la región latinoamericana y el Caribe están enfrentándose a la pandemia desde una posición más débil que el resto del mundo y los efectos más graves que vivirán según la CEPAL son: una reducción del comercio internacional, la caída de los precios de los productos primarios, la intensificación de la aversión al riesgo y el empeoramiento de las condiciones financieras mundiales, una menor demanda de servicios turísticos y una reducción de las remesas. Si bien esta situación afectará a toda la región, es aún más preocupante para el $54 \%$ de la población total que subsiste a través del trabajo informal. Según la Organización Internacional del Trabajo, el 90\% de este sector sufrirá perdidas masivas en sus ingresos, esto debido a suspensión de salarios, así como la pérdida definitiva de sus trabajos. Esto se traduce en al menos 140 millones de personas que ahora se verán más cerca de la pobreza y la desigualdad.

A raíz del gran reto al que se enfrenta América Latina, es importante iniciar a configurar estrategias que se puedan implementar para mejorar la situación actual, especialmente estrategias para el apoyo de los más vulnerables y afectados por la pandemia. Desde el Instituto de Investigación y Estudios Superiores en Arquitectura y Diseño (INDIS) de la Universidad Rafael Landívar se desarrollan programas de investigación e incidencia en temas relacionados al diseño en todas sus formas. Dentro de sus programas se encuentra el Centro de Atención a la Mipyme (CAM), el cual está destinado a desarrollar proyectos que empoderen a las mipymes locales por medio del diseño y la innovación. Uno de los proyectos principales del centro es la investigación de tendencias globales, estas se transforman en contenido práctico que pueda utilizar el sector de productos hechos a mano y de manufactura ligera para el desarrollo de propuestas de valor alineados a las necesidades del consumidor. Es aquí de donde surge la invitación a impulsar y convocar proyectos que enlacen la disciplina del diseño y el producto hecho a mano de la región, llevando a un nuevo nivel este sector en riesgo. La idea es unir las técnicas ancestrales desde las manos de los portadores de este conocimiento con la visión, experiencia y formación que tienen los diseñadores.

\section{La artesanía y los retos que enfrenta en Latinoamérica}

Para comprender la propuesta, se debe conocer lo que engloba el término artesanía, también conocida como producto hecho a mano. Debido a la gran cantidad de técnicas y productos y procesos que se utilizan para el desarrollo de las artesanías, la UNESCO desarrolló una descripción que sirve como base para su caracterización ante el mercado internacional. Ellos definen artesanía como: 
Los productos artesanales son los producidos por artesanos, ya sea totalmente a mano, o con la ayuda de herramientas manuales o incluso de medios mecánicos, siempre que la contribución manual directa del artesano siga siendo el componente más importante del producto acabado. Se producen sin limitación por lo que refiere a la cantidad y utilizando materias primas procedentes de recursos sostenibles. La naturaleza especial de los productos artesanales se basa en sus características distintivas, que pueden ser utilitarias, estéticas, artísticas, creativas, vinculadas a la cultura, decorativas, funcionales, tradicionales, simbólicas y significativas religiosa y socialmente (UNESCO, 1997).

Además de esta definición, sería ideal brindar el contexto actual del sector involucrado, pero aquí es necesario resaltar que actualmente se encuentran pocos recopilados sobre la artesanía latinoamericana y sus productores. Esto desde ya se presenta como un reto para el sector, pues sin conocer las dimensiones de la población que la conforman es difícil desarrollar estrategias regionales de apoyo y seguimiento. Los datos de esta índole existen para cada país y son manejados por distintas organizaciones conformadas localmente, y manejan la información bajo sus propias disposiciones. Uno de los estudios que se encuentran en la red que incluye a varios países es la titulada Caracterización del Sector Artesanal Latinoamericano realizado desde Colombia y coordinado por Adriana Uribe durante el 2011. Este estudio incluye información sobre siete países que entonces conformaban la ahora inexistente Red Iberoamericana de Innovación y Transferencia para el Fortalecimiento Artesanal (RIFTA), del Programa Iberoamericano de Ciencia y Tecnología para el Desarrollo (CYTED). Esta publicación describe la realidad de diferentes comunidades artesanas de los países involucrados a través de una encuesta realizada en cada una de estas comunidades. Sin embargo, no menciona la realidad económica bajo la que viven ni la calidad de vida que llevan. Uno de los indicadores que se logra encontrar sobre calidad de vida es la mención que hace Herrera que, a diferencia de los países del norte del planeta, en Latinoamérica la artesanía suele darse principalmente en la periferia de las ciudades, en donde viven enfrentándose a múltiples condiciones adversas (Herrera, 2016).

Varios autores expresan el potencial que el sector artesanal latinoamericano tiene para impulsar el desarrollo económico y social de sus países. Silvia Navarro expresa que a través de este sector se pueden lograr fuentes de empleos directos e indirectos, además impulsando este sector al punto de lograr fuentes de ingresos más atractivos para los productores se podría lograr evitar la migración a las zonas urbanas de los países. Otro posible beneficio es el empoderamiento femenino, pues al hacer crecer este sector se podrían abrir más espacios para que las mujeres participen en las labores y generen sus propios ingresos (Hoyos, 2013).

A partir de la publicación de Adriana Uribe se logran concluir varios retos al que el sector ya se enfrentaba desde entonces. Entre los hallazgos de esta publicación uno de los más relevantes es el de cómo los artesanos consideran que los diseños exclusivos suelen ser la ventaja competitiva más importante que ellos puedan tener, siendo mencionada por el 79.5\% de los artesanos encuestados (Uribe, 2011). Esta afirmación es importante pues las mismas comunidades ya han logrado detectar oportunidades que podrían impulsarlas. Sin embargo, se enfrentan con el reto de desarrollar y llevar a cabo estrategias de este tipo. 
Otro hallazgo importante encontrado en la publicación de Uribe describe cómo un cuarto de los artesanos no ha involucrado a sus hijos en la labor que desempeña. Esto sucede principalmente por iniciativa de los padres pues ellos mismos tienen la percepción de que a través de su labor generan bajos ingresos, además consideran que su oficio es poco valorado, no solo por el mercado, incluso por actores de la misma cadena productiva. Esta estadística contrasta con el hecho que el $44.9 \%$ de los participantes mencionaron haber aprendido su labor de sus padres, por lo cual se podría prever que con la siguiente generación esta estadística se vería reducida considerablemente. Los retos que estos hallazgos implican se relacionan con la perdida del conocimiento ancestral y el patrimonio inmaterial que resguardan los maestros artesanos y que de no ser aprendidos por las generaciones más jóvenes se podrían perder pronto. La UNESCO estableció en 2005, en la Convención sobre la Protección y Promoción de la Diversidad de las Expresiones Culturales, que la diversidad cultural es de las mayores riquezas humanas y este es un patrimonio que debe resguardarse pues son importantes para la identidad cultural y para la salvaguarda de la diversidad cultural y la creatividad humana. Fue así como desde entonces trataron de promover el resguardo de estos conocimientos y la importancia del diálogo entre culturas para fomentar la interculturalidad y la unión entre sectores (Benítez Aranda, 2007).

Por otra parte, otro desafío importante al que los artesanos y las organizaciones que les apoyan se enfrentan es el lograr desmentir ciertas creencias y generalizaciones que la sociedad, principalmente la local, tiene del sector. El principal paradigma que se debe romper es el de la creencia que el producto artesanal es sinónimo de souvenir, producto decorativo o de bajo costo. Esto se ve ligado a la reproducción de objetos con diseños replicados de la artesanía local pero manufacturados fuera, a gran escala y por industrias que logran minimizar los costos a una fracción del real. Asimismo, Herrera (2016) también menciona que otra limitante para este sector es el difícil acceso a plataformas digitales, principalmente por su ubicación geográfica y por la distinción social. El autor afirma que el nivel tecnológico aplicado a productos y procesos artesanales es limitado y esto a su vez limita los alcances de los diseño. Cabe resaltar que, a pesar de que a muchos les pasa, esto no aplica para todos, pues hay artesanos que incluso cuentan con estudios universitarios, lo cual les facilita el acceso a más recursos y a distintas alianzas.

\section{El diseñador y sus capacidades}

Al tratar de mencionar los posibles personajes capaces de tener un impacto positivo para el desarrollo del sector artesanal latinoamericano, es inevitable mencionar a los diseñadores industriales. La Organización Mundial del Diseño (WDO) define actualmente el diseño industrial como:

El diseño industrial es un proceso estratégico de resolución de problemas que impulsa la innovación, construye el éxito empresarial y conduce a una mejor calidad de vida a través de productos, sistemas, servicios y experiencias innovadores (World Design Organization, 2020). 
Esta misma organización define al diseñador industrial como:

Los diseñadores industriales colocan al ser humano en el centro del proceso. Adquieren una comprensión profunda de las necesidades del usuario a través de la empatía y aplican un proceso pragmático de resolución de problemas centrado en el usuario para diseñar productos, sistemas, servicios y experiencias. Son partes interesadas estratégicas en el proceso de innovación y están en una posición única para unir diversas disciplinas profesionales e intereses comerciales. Valoran el impacto económico, social y ambiental de su trabajo y su contribución a la creación conjunta de una mejor calidad de vida (World Design Organization, 2020).

Es fácil comprender a través de estas definiciones el potencial que tiene el diseño industrial para generar proyectos en conjunto con las comunidades artesanas. El diseñador tiene la habilidad y la formación ideal para poder comprender lo que vive el artesano, sus procesos y alcances; y luego poder conectarlo con lo que necesita el consumidor para generar propuestas de valor que logren penetrar el mercado de forma efectiva y generen un impacto positivo en el productor. La experiencia y formación por la que pasan los diseñadores los preparan para este tipo de proyectos de alto impacto. Entre las habilidades principales con las que cuentan se pueden enlistar: la capacidad de investigar y analizar el mercado para determinar las estrategias ideales para el desarrollo de un proyecto; la capacidad de conectarse y comprender a los actores involucrados en el proceso, desde el productor hasta el consumidor; el conocimiento de tendencias y consideraciones estéticas a tener en cuenta; y la habilidad de iterar dentro de su mismo proceso para desarrollar una propuesta de valor que beneficie a todas las partes interesadas. Sin embargo, este tipo de habilidades no es exclusivo de los diseñadores industriales, es común encontrar actores de diferentes disciplinas con características aplicables, especialmente aquellos que desempeñan labores creativas como los arquitectos.

\section{La unión de dos fuerzas: la artesanía contemporánea}

Los artesanos que se dedican a la misma labor suelen producir y reproducir los mismos diseños, pues es lo que han aprendido y elaborado desde el inicio. Son pocos aquellos que desarrollan propuestas diferentes a lo tradicional. Es aquí en donde deben involucrarse los diseñadores, el potencial de generar proyectos que concluyan con propuestas diferenciadoras para las comunidades involucradas debe ser aprovechado lo más pronto posible. Este tipo de proyectos son los que conforman la artesanía contemporánea, también llamada neo-artesanía. La sociedad Artesanías de Colombia define la artesanía contemporánea como:

Es la producción de objetos útiles y estéticos desde el marco de los oficios, en cuyos procesos se sincretizan elementos técnicos y formales procedentes 
de otros contextos socioculturales y otros niveles tecnoeconómicos, tiene una característica de transición hacia la tecnología moderna y/o la aplicación de principios estéticos de tendencia universal y/o académicos y tiende a destacar la creatividad individual expresada por la calidad y originalidad del estilo (Artesanías de Colombia, 2020).

Actualmente ya se cuentan con diversas propuestas que utilizan este nuevo modelo de creación de productos artesanales en diferentes regiones del mundo. Desde competencias de diseños innovadores bajo este concepto, hasta asociaciones dedicadas al impulso de esta nueva versión de las labores tradicionales. En España, por ejemplo, se cuenta con la Sociedad de Artesanos Contemporáneos (SACo) a través de la cual se han llevado a cabo colecciones de productos de ocho oficios distintos. Entre sus técnicas se encuentran las más frecuentes como madera y cuero, hasta oficios especializados como la fabricación de sombreros y una especialista en el arte plumaria. Es importante resaltar que la artesanía contemporánea se está planteando ante el mercado global como un producto de lujo y así lo perciben los consumidores, a diferencia de la percepción que se tiene de la artesanía tradicional. Sin embargo, también se debe mencionar que para lograr posicionarse de esta manera, los productores deben tener especial cuidado durante todo el proceso, poniendo mucha atención al detalle de los acabados, los materiales a utilizar y su manejo.

Por otra parte, Herrera (2016) describe en su trabajo varios casos de estudio que describen proyectos que entrelazaban la artesanía y la fabricación digital. El menciona la iniciativa Fab Academy creada por el MIT y el Institud d'Arquitectura Avançada de Catalunya en el 2009. Bajo esta iniciativa, durante el 2012 se desarrolló un telar con la premisa de que fuera una propuesta que redujera los costos y tiempos de producción. Fue así como el arquitecto Walter González, tras aprender sobre los telares, logró desarrollar una propuesta de telar andino fabricado en un fablab en tan solo una hora y con un costo de alrededor de \$100 (Herrera, 2016). Además, esta propuesta fue inscrita como modelo de utilidad en Perú y desde entonces cuenta con el registro necesario, lo cuál formaliza aún más estos proyectos.

\section{Las soluciones que brinda la artesanía contemporánea}

Esta evolución del sector trae consigo oportunidades de mejora y crecimiento para las comunidades artesanas de la región. Esta misma evolución se hace notar cuando se utilizan los términos adecuados, pues estos productos ya no son sólo artesanías, son artesanías contemporáneas que nacen de la sincronía entre el diseño y el oficio.

El primer reto mencionado anteriormente hablaba sobre la falta de diseños exclusivos para ser producidos y comercializados por los artesanos. Luego de comprender la labor del diseñador, no es difícil figurarse cómo la artesanía contemporánea tiene el potencial de generar un impacto positivo en este ámbito. El desarrollo de propuestas innovadoras que utilice los procesos tradicionales pero que presente formas y funciones no antes vistas es la vía de acción que la artesanía contemporánea abre ante esta problemática. A través de este tipo de proyectos y colaboraciones, se le da al artesano un producto diferenciador 
y con el valor agregado de la unión de estas dos fuerzas. Estas propuestas además abren la posibilidad de abrirse a nuevos mercados y sectores económicos. Sin embargo, para lograr esto, es importante resaltar que el éxito de estas colaboraciones radica en el respeto de ambas partes y la comunicación efectiva. Al conocer las capacidades, habilidades y técnicas de ambas partes, los límites bajo los que se tienen que regir las propuestas son solamente los definidos por el material. Cuando un diseñador logra conocer cómo trabaja su contraparte, puede desarrollar ideas innovadoras pero realizables. De igual forma, cuando un artesano comprende la visión del diseñador, puede brindar sus propios aportes y alternativas que logren el mayor alcance a las ideas propuestas.

A través de esta primera línea de acción propuesta, también se podría tener un impacto positivo para la segunda problemática planteada anteriormente, la cual habla sobre cómo se han dejado de transmitir los oficios a las nuevas generaciones por el poco valor que se le da a los productos que realizan los artesanos. Al impulsar proyectos que generen nuevas colecciones que entren en el mercado de lujo se puede lograr mejorar los ingresos de las comunidades involucradas, agregándole valor a sus oficios. Esto podría revertir el pensamiento de los artesanos actuales, logrando que trasladen sus conocimientos a las nuevas generaciones, bajo la seguridad de poder brindarles una mejor calidad de vida. Como beneficio paralelo, también se rescataría el conocimiento ancestral y se resguardaría en las comunidades, asegurando el patrimonio intangible de la región.

Por último, bajo esta misma línea de acción, también se puede abordar el concepto equivocado que se tiene regionalmente sobre los oficios y los productos artesanales. Esta idea errónea se podrá desmentir a través de la demostración. Generar propuestas de lujo, atractivas e innovadoras podrá llamar la atención local y devolverle el prestigio que se merece esta labor. Las colecciones limitadas son una estrategia importante al llevar a cabo este tipo de proyectos. Limitar las cantidades apela a la necesidad del consumidor de sentirse único, pues sabe que contará con productos que se producirán en una sola serie y no serán de acceso para nadie más una vez se acaben, haciendo que valga la pena invertir más por sus piezas. Además, esto también ayuda a controlar la calidad de los productos, pues los esfuerzos de los productores se concentrarán en la colección a desarrollar y no agotarán sus esfuerzos. Una segunda línea de acción importante que se debería de considerar, pero más compleja en cuanto a realización, es la de la creación de redes y comunidades que promuevan la artesanía contemporánea regionalmente. La RITFA tenía un enfoque alineado a lo que aquí se propone. Su red incluyó a México, El Salvador, República Dominicana, Colombia, Perú, Argentina, Chile y Venezuela. Durante alrededor de cinco años realizaron proyectos centrados en el sector. Entre sus aportes se incluyen la generación de conocimiento sobre el sector en la región, promoción de la formalización del sector ante gobiernos locales, le dieron mayor visibilidad a la artesanía y coordinaron el impulso de distintos beneficiados en ferias y colaboraciones. En cuanto al impacto directo para los artesanos, mencionan haber llevado a cabo capacitaciones que mejoraron las habilidades técnicas, administrativas, comerciales y de mercadeo de los participantes (CYTED, 2020). Sin embargo, no hay más documentación sobre esta red pasado el 2015, cuando parece que se disolvió. Es innegable la magnitud de este proyecto, así como lo son sus resultados. Con la pandemia aun amenazando al continente, es ideal considerar este tipo de organizaciones para la recuperación e impulso del sector, el cual ya era vulnerable previo a la crisis. 


\section{Consideración: El respeto}

Al hablar sobre la primera propuesta de acción, se menciona brevemente la importancia del respeto y la comunicación entre el artesano y el diseñador para el desarrollo de proyectos de artesanía contemporánea. Sin embargo, se considera de suma importancia hacer énfasis en esta premisa. Es gracias a esta relación de iguales que es posible el desarrollo de la artesanía contemporánea. El proceso creativo en estos proyectos se ve enriquecido por las diferencias entre los actores.

\section{Las oportunidades que brinda la pandemia}

La crisis sanitaria que el COVID-19 está generado cambios importantes en las necesidades de los consumidores. Por consecuencia, también en los patrones de tendencias que se estaban viendo, principalmente causando el fortalecimiento de varios movimientos. Estos cambios deben ser considerados por todos los involucrados en los sectores de producción incluyendo a la artesanía contemporánea para poder brindar soluciones acertadas. Una de las tendencias que ya estaban identificadas y venía en crecimiento era la de los productos sustentable y con bajo impacto ambiental. A partir de la pandemia, esta tendencia es más clara e importante que antes y se viene con mayor fuerza. Esto era un tema que se venía popularizando hace tiempo, sin embargo, ahora será exigido por el mercado. La sustentabilidad ya no se hablará tan solo como una tendencia de moda, ahora sí se asimilará por consumidores y será un punto de partida en el desarrollo de proyectos. Esto implica una mayor atención por parte del mercado a todo el proceso de producción de un producto, desde la obtención de los materiales, pasando por sus procesos y llegando a considerar hasta el método de entrega y su empaque. Anteriormente, era difícil hacerle competencia a los productos fabricados en masa por los costos que estos manejaban, sin embargo, la sustentabilidad a partir de ahora será una parte importante de la propuesta de valor de los productos y podrá hacerles frente a los precios bajos de los productos masificados.

Conectado con el impacto ambiental, otro movimiento que se refuerza a partir de la crisis es el impacto social que un producto, marca o empresa tiene. Condiciones de trabajo dignas, remuneraciones económicas justas y responsabilidad social empresarial tendrán mayor importancia ante el consumidor y su toma de decisiones al elegir un producto. Esto se verá como una ventaja para la artesanía contemporánea, sin embargo, es importante crear una estrategia sólida que aproveche las características de los productos hechos a mano. Estrategias como incluir la historia de los fabricantes o de las comunidades artesanas han resultado efectivas previo a la pandemia, es ahora cuando se pueden aprovechar aún más estas historias. Al utilizar este tipo de marketing, es importante considerar que el solo hecho de ser productos hechos a mano no logrará vender si estos resultan mal acabados, hay que tener claro que la calidad y funcionalidad de una propuesta son los factores principales bajo los que los consumidores toman decisiones.

Por otra parte, el cierre de fronteras y las restricciones comerciales que la mayoría de los países han implementado para evitar la propagación del virus han ocasionado múltiples inconvenientes debido a las limitadas importaciones que se están llevando a cabo, hacién- 
doles reconsiderar la dependencia que tenían del mercado extranjero. Esto resulta en una reconsideración del mercado y de la industria local. Esto se presenta como una oportunidad ideal para el sector artesanal, pues desde ya los consumidores están dirigiendo su interés a las empresas nacionales, no solo por ser las únicas que pueden brindarles servicios sin tantas restricciones, también para apoyarlas durante esta crisis. Este interés permanecerá aún después de la pandemia y es entonces cuando la artesanía contemporánea deberá esforzarse por hacerse ver a través de propuestas innovadoras y de calidad, demostrando que a través de los oficios se puede hacer mucho más que artículos de decoración y souvenirs.

\section{Otras tendencias}

Conocer las tendencias que tienen más fuerza durante la crisis y comprender lo que el consumidor necesita es clave para lograr adaptarse y proponer productos que satisfagan sus necesidades y anhelos. El confinamiento al que una gran parte de la población se enfrentó, trajo consigo nuevas necesidades, y por ahora todo gira en torno al hogar. La casa se ha vuelto el centro de nuestras vidas y son más las tareas que se deben realizar desde la misma. Es por esto que ahora el mercado de productos para el hogar es más fuerte que nunca. Hay muchos productos que se encuentran en casa que nunca terminaron de convencer al usuario, así como hay otros que no se alinean a lo que el mismo quiero transmitir desde su hogar, provocando la necesidad de hacer cambios al respecto. Además, todas las nuevas actividades que se están realizando desde el hogar crean nuevas necesidades y oportunidades que antes no se presentaban. Nuevas necesidades también surgen a raíz de los espacios de la casa, pues estos han tenido que adaptarse a las nuevas actividades también, ya sean espacios con múltiples funciones o espacios designados a actividades específicas. Estas tendencias se describen un poco mejor a continuación:

\section{El home office y el home school}

Una tendencia importante que se venía hablando anteriormente pero que ahora tuvo una implementación radical fue la del trabajo en casa. La necesidad de adaptarse en un período muy corto al teletrabajo trajo la necesidad de incluir un espacio adecuado en casa, así como conseguir las herramientas necesarias para poder llevar a cabo efectivamente las tareas del trabajo. Además, ahora que los hijos deben llevar a cabo sus estudios de forma remota también, es necesario adaptarles espacios adecuados en el que puedan realizar sus tareas y recibir sus clases. Es importante establecer los espacios y separarlos, en la medida de lo posible, de los espacios de descanso y entretenimiento para que no se vean abrumados ante la limitación de movilidad a la que nos enfrentamos.

\section{Orden}

Una de las principales actividades en las que se ha utilizado el tiempo libre, es la limpieza y organización del hogar, incluyendo espacios y accesorios que no habían sido considerados 
antes. El objetivo de las personas es crear un ambiente acogedor y que se alinee a sus personalidades. Es así como ya no hay espacio para accesorios que no llenen las expectativas. A raíz de esto, se ven con la necesidad de cambiar y adquirir productos que sean más de su agrado. Además que se ha aumentado la necesidad de contar con accesorios que ayuden a mantener más ordenado y mejor clasificado todo dentro de la casa.

\section{Naturaleza}

Ahora que muchas personas se ven en confinamiento, la naturaleza es algo que se añora en gran medida. Es por esto que las propuestas que incluyen plantas o hacen referencia a este tema se ven con más potencial. El ideal es lograr crear propuestas que logren llevar plantas fáciles de mantener a casa. Estas propuestas no deben perder el objetivo de alinearse a los gustos del consumidor, es por esto que siempre es importante conocer el grupo objetivo. Aquí además se pueden aprovechar los beneficios atribuidos a las diferentes especies de plantas. Esto puede ser aprovechado en las estrategias de mercadeo.

\section{Limpieza}

Es importante considerar que una tendencia que surge a raíz de la pandemia son los productos y materiales fáciles de limpiar. La conciencia colectiva que se desarrolló ante la higiene será algo que se quedará en las personas por un tiempo, es por esto que las propuestas que se desarrollen posterior a la pandemia deberán considerar esto como requisito en el proyecto. Esto aplica en productos para el hogar como en accesorios portátiles. Hacer énfasis en las cualidades higiénicas o de limpieza que un producto pueda presentar se podrá presentar como ventaja competitiva.

\section{Conclusiones}

El diseño industrial es una disciplina que se caracteriza por su capacidad de adaptación, pudiendo intervenir en muchos campos y aliarse con múltiples disciplinas. Es por esto por lo que son actores fundamentales en la revitalización de la artesanía latinoamericana. Su visión global y su comprensión de lo local tienen el potencial de generar propuestas que cambien el rumbo del sector artesanal. Esto, toma aún más relevancia considerando la pandemia, pues las comunidades artesanas suelen ser comunidades vulnerables y la crisis tendrá un mayor impacto económico y social en ellas. Aunar el sector artesanal con el diseño puede llegar a rescatar el conocimiento ancestral único con el que cuenta la región, así como puede ayudar a mejorar la realidad de las personas que se involucren.

Es papel de las autoridades locales impulsar estos proyectos y alianzas, incluyendo gobiernos y universidades que tengan la capacidad de involucrarse. Desde la Universidad Rafael Landívar se han implementado proyectos de intervención a pequeños artesanos por parte de estudiantes de diseño. Ha demostrado ser una plataforma con potencial, pues no solo los artesanos se benefician de estas colaboraciones al contar con nuevas líneas de diseño 
únicas, también los estudiantes se han visto sumamente beneficiados pues lo que aprenden durante el proceso y la experiencia que se llevan es algo que a través de la teoría no se puede transmitir.

Es ahora, cuando se debe buscar implementar e impulsar a los más vulnerables y es nuestra responsabilidad social crear espacios e impulsar proyectos que incluyan a los más vulnerables.

\section{Listado de referencias bibliográficas}

Artesanías de Colombia. (5 de junio de 2020). Artesanía contemporánea o neoartesanía. Obtenido de Artesanías de Colombia: http://artesaniasdecolombia.com.co/PortalAC/ GlosarioPalabra/artesania-contemporanea-o-neoartesania_48

BBC. (9 de abril de 2020). Coronavirus: las oscuras proyecciones del FMI sobre la economía mundial por los efectos del coronavirus. Obtenido de BBC News Mundo: https://www. bbc.com/mundo/noticias-internacional-52225918

Benítez Aranda, S. (2007). La artesanía latinoamericana como factor de desarrollo económico, social y cultural: a la luz de los nuevos conceptos de cultura y desarrollo. Obtenido de http:// www2.congreso.gob.pe/sicr/cendocbib/con5_uibd.nsf/48D7253265028FB40525827A00 61790C/\$FILE/CyD_LaArtesaniaLatinoamericana.pdf

CEPAL. (21 de abril de 2020). Pandemia del COVID-19 llevará a la mayor contracción de la actividad económica en la historia de la región: caerá -5,3\% en 2020. Obtenido de Comisión Económica para América Latina y el Caribe: https://www.cepal.org/es/comunicados/ pandemia-covid-19-llevara-la-mayor-contraccion-la-actividad-economica-la-historia-la

CYTED. (2020). Fortalecimiento de la industria artesanal. Obtenido de Programa Iberoamericano de Ciencia y Tecnología para el Desarrollo (CYTED): http://www.cyted.org/ es/destacada_ritfa

Forbes. (16 de abril de 2020). FMI: COVID-19 puede provocar "otra década perdida" en Lationamérica. Obtenido de Forbes Centroamérica: https://forbescentroamerica. com/2020/04/16/fmi-covid-19-puede-provocar-otra-decada-perdida-en-latinoamerica/

Herrera, P. (11 de noviembre de 2016). Artesanía en Latinoamérica: Experiencias en el contexto de la Fabricación Digital. Obtenido de http://papers.cumincad.org/data/works/ att/sigradi2016_814.pdf

Hoyos, S. N. (junio de 2013). Artesanía latinoamericana: folklor y comercio. Obtenido de Manos de Artesano: https://manosdeartesano.files.wordpress.com/2013/06/silvananavarro-hoyos.pdf

UNESCO. (6 de octubre de 1997). Artesanía y Diseño. Obtenido de Organización de las Naciones Unida para la Educación, la Ciencia y la Cultura: http://www.unesco.org/new/ es/culture/themes/creativity/creative-industries/crafts-and-design/

Uribe, A. (Diciembre de 2011). Libros y Publicaciones. Obtenido de Dr. León Cabello Cervantes: https://drleoncabello.wordpress.com/libros-publicados/

World Design Organization. (2020). Definition of industrial design [Definición del diseño industrial]. Obtenido de World Design Organization: https://wdo.org/about/definition/ 


\begin{abstract}
Products that include artisan techniques in their production processes are becoming increasingly popular, not only for the experience they provide the consumer, but also for the growing social and environmental awareness that is booming and that motivates the purchase of proposals with the value that only handmade products can provide. This presents an invaluable opportunity for all the master craftsmen that Latin America has. Although it is true that some time ago their ancestral techniques were lost, it is now when the ideal opportunity for their recovery presents itself. However, there are knowledge and strategies of difficult access for these communities of artisans, which if well implemented can considerably increase the competitiveness of the products. This knowledge can be found through designers and researchers, who have access, training and the ability to transfer information to artisans so that the proposals generated are of even greater value, with the potential to penetrate the market. global.
\end{abstract}

Keywords: Design - Crafts - Collaborative design - Local - Global.

Resumo: Os produtos que incluem técnicas artesanais em seus processos de produção estão se tornando cada vez mais populares, não apenas pela experiência que fornecem ao consumidor, mas também pela crescente conscientização social e ambiental que está crescendo e que motiva a compra de propostas com o valor que somente produtos artesanais podem oferecer. Isso representa uma oportunidade inestimável para todos os mestres artesãos da América Latina. Embora seja verdade que há algum tempo suas técnicas ancestrais foram perdidas, é agora que a oportunidade ideal para sua recuperação se apresenta. No entanto, existem conhecimentos e estratégias de difícil acesso para essas comunidades de artesãos que, se bem implementados, podem aumentar consideravelmente a competitividade dos produtos. Esse conhecimento pode ser encontrado por designers e pesquisadores, que têm acesso, treinamento e capacidade de transferir informações para artesãos, para que as propostas geradas sejam de valor ainda maior, com potencial de penetração no mercado. global.

Palavras chave: Design - Artesanato - Design colaborativo - Local - Global.

[Las traducciones de los abstracts fueron supervisadas por el autor de cada artículo] 